\title{
Diversité et structure de la végétation ligneuse dans la ville de Malanville au Nord-Bénin
}

\author{
Baké OROU WARI ${ }^{1 *}$, Soufouyane ZAKARI ${ }^{1}$, Mama DJAOUGA ${ }^{1}$, \\ Ismaïla TOKO IMOROU ${ }^{1}$, Ibouraïma YABI ${ }^{2}$, Brice A. H. TENTE ${ }^{3}$ et Julien G. DJEGO ${ }^{4}$ \\ ${ }^{1}$ Laboratoire de Cartographie, (LaCarto), Université d'Abomey-Calavi, 10 BP 1082 Cotonou, Cadjèhoun, \\ Bénin. \\ ${ }^{2}$ Laboratoire Pierre PAGNEY, "Climat, Eau, Ecosystèmes et Développement" (LACEEDE), Université \\ d'Abomey-Calavi, BP 922, Abomey-calavi, Bénin. \\ ${ }^{3}$ Laboratoire de Biogéographie et d'Expertise Environnementale (LABEE), Université d'Abomey-Calavi, BP \\ 677 Abomey-Calavi, Bénin. \\ ${ }^{4}$ Laboratoire d'Écologie Appliquée (LEA/FSA/UAC), 01 BP 526 Cotonou, Bénin. \\ *Auteur correspondant ; E-mail : waribake@ gmail.com ; Tel. : + 22997255438.
}

\section{REMERCIEMENTS}

Le présent travail a été réalisé grâce à l'appui financier du projet Cartographie et modélisation $d u$ carbone séquestré par la végétation en milieu urbain pour l'atténuation des changements climatiques et la promotion des villes durables au Bénin, (Projet MOVIC) du Programme Fonds Compétitifs de Recherche (PFCRIII) de l'Université d'Abomey-Calavi. Les auteurs remercient l'Université d'Abomey-Calavi et tous les initiateurs de ce programme.

\begin{tabular}{rll}
\hline Received: 24-08-2020 & Accepted: 22-01-2021 & Published: 28-02-2021 \\
\hline
\end{tabular}

La végétation urbaine a le potentiel de relever de nombreux défis environnementaux liés à la durabilité des villes. L'objectif de cette étude était de caractériser la végétation ligneuse de Malanville. La méthode de relevé phytosociologique est utilisée pour la collecte des données. L'échantillonnage aléatoire stratifié a permis d'installer 300 placeaux carrés de 1 ha, générés au hasard par l'outil «Data Management » du logiciel ArcGIS 10.5, suite à un maillage de l'armature urbaine. Le travail a permis de dénombrer 68 espèces ligneuses réparties 58 genres et 33 familles. La diversité floristique est relativement faible et varie de façon significative $(\mathrm{p}<0,05)$ selon les unités d'occupation des terres (richesse spécifique : 2,75 à 6,75; diversité de Shannon : 0,83 à 1,64 bits ; équitabilité de Pielou : 0,48 à 0,63). Les paramètres de structure décroissent significativement $(\mathrm{p}<0,01)$ selon les unités d'occupation des terres (densité moyenne : 6,56 à 59,25 N/ha ; surface terrière : 0,77 à 4,52 $\mathrm{m}^{2} /$ ha ; circonférence moyenne : 90,7 à $121,17 \mathrm{~cm}$ ). La ville de Malanville regorge d'une importante biodiversité floristique constituée à $61 \%$ d'espèces exotiques. Dans la recherche de solutions locales aux répercussions des changements climatiques, cette étude est un atout pour la conservation de la végétation ligneuse urbaine permettant de construire des villes plus durables, attractives et vertes.

(C) 2021 International Formulae Group. All rights reserved.

Mots clés : Diversité floristique, structure de la végétation, végétation en milieu urbain, Malanville, Bénin. 


\title{
Diversity and structure of woody vegetation in the town of Malanville in Northern Benin
}

\begin{abstract}
Urban vegetation has the potential to meet many challenges environmental issues related to the sustainability of cities. The objective of this study was to characterize the woody vegetation of Malanville. The phytosociological survey method was used for data collection. Stratified random sampling allowed the installation of 300 square plots of 1 ha, randomly generated by the "Data Management" tool of the ArcGIS 10.5 software, following a meshing of the urban framework. The work enabled the enumeration of 68 woody species distributed in 58 genera and 33 families. The floristic diversity is relatively low and varies significantly ( $p<$ 0.05 ) according to land use units (specific richness: 2.75 to 6.75 ; Shannon's diversity: 0.83 to 1.64 bits; Pielou's equitability: 0.48 to 0.63 ). The structure parameters decrease significantly $(\mathrm{p}<0.01)$ according to the land use units (mean density: 6.56 to $59.25 \mathrm{~N} / \mathrm{ha}$; basal area: 0.77 to $4.52 \mathrm{~m} / \mathrm{ha}$; mean circumference: 90.7 to $121.17 \mathrm{~cm}$ ). The town of Malanville has an important floristic biodiversity, $61 \%$ of which is made up of exotic species. In the search for local solutions to the impacts of climate change, this study is an asset for the conservation of urban woody vegetation, enabling the construction of more sustainable, attractive and greener cities.
\end{abstract}

(C) 2021 International Formulae Group. All rights reserved.

Keywords : Floristic diversity, vegetation structure, urban vegetation, Malanville, Benin.

\section{INTRODUCTION}

À partir des années 1990, les scientifiques ont commencé par s'interroger sur la place du milieu urbain et de ses espaces à caractère naturel (connus sous le nom d'espaces végétalisés urbains) dans la protection, la valorisation de la biodiversité et l'optimisation des services écosystémiques (Selmi et Weber, 2017). Ainsi, les villes sont de grands centres de demande de services écosystémiques ainsi que des sources d'effets nocifs sur l'environnement. Elles font parties des écosystèmes les plus fortement impactés par les activités humaines. Par ailleurs l'accroissement démographique des villes et la dégradation des ressources naturelles, sont des phénomènes intimement liés. L'accélération sans précédent du développement urbain découlant de l'accroissement démographique a considérablement affecté les rapports de l'Homme avec les arbres et les forêts (FAO, 2012). En effet, dans le souci de satisfaire leur besoin, les hommes détruisent dans leur environnement immédiat et même lointain des sites de biodiversité de grandes valeurs écologiques. Face à cette situation un regain d'intérêt de la part des gestionnaires urbains, s'est récemment fait sentir et se traduit de manière emblématique par la place de plus en plus forte accordée au végétal en ville (Blanchart et al., 2017). L'avènement du concept de développement durable dans les dernières décennies du $\mathrm{XX}^{\mathrm{e}}$ siècle, a donné une nouvelle impulsion et présente la nature, vue ici à travers le végétal, comme une possible réponse aux impacts négatifs de la ville (Nowak et al., 2006 ; Matusoka et al., 2008). Les gestionnaires urbains doivent prendre conscience de l'existence d'un patrimoine naturel placé sous leur responsabilité, qu'il convient de connaître, gérer et conserver dans les meilleures conditions possibles. Au-delà d'une importante diversité, comme les écosystèmes naturels, les écosystèmes urbains assurent la régulation du microclimat en créant des îlots de fraicheur, à travers le stockage de carbone (Yuanying et al., 2008) dans sa biomasse aérienne, l'évapotranspiration et la projection au sol de leur ombrage. Toutefois, chaque communauté végétale, accumule la biomasse ligneuse suivant sa composition floristique et sa structure (Henry et al., 2010 ; Djomo et al., 2010). Une meilleure connaissance de l'état de la végétation urbaine est nécessaire pour sa conservation afin d'optimiser les différents services écosystémiques rendus aux citadins. 
Dans les grandes villes africaines en général et celles du Bénin en particulier, la végétation urbaine a fait l'objet de plusieurs recherches (Lougbegnon, 2013 ; Osséni et al., 2014 ; Amontcha et al., 2017). Ces recherches ont surtout étudié la végétation urbaine à travers les indicateurs tels que la composition floristique, la richesse spécifique et la structure. Les différents résultats obtenus dans la littérature ont révélé les potentialités de la végétation urbaine au Bénin dont l'importance n'est plus à démontrer, dans l'atténuation de certains effets négatifs des changements climatiques en cours. Aucune étude, à notre connaissance, n'a pas évalué le cortège végétatif de la ville de Malanville. Or, au Bénin, Malanville fait partie de l'une des Communes les plus vulnérables au changement climatique en témoignent les dégâts causés par les inondations en 2013 dont la perte de valeurs de production est estimée à 15.801.080 Fcfa (PNUD, 2019). En plus, de par sa position géographique, la ville de Malanville subie la rigueur du climat sahélien qui rend sa végétation de plus en plus vulnérable. Le choix de la ville de Malanville se justifie non seulement par sa vulnérabilité aux changements climatiques, mais aussi par le rôle important que joue la végétation ligneuse urbaine pour le bien être des citadins. En effet, l'amplitude très élevée de température enregistrée dans la ville, fait de l'arbre le seul endroit propice pour le repos pendant la chaleur au détriment des bâtis. Aussi, la végétation peut atténuer les effets de l'harmattan intense qui sévit dans la ville de novembre à mars. A tout cela, s'ajoute des défis environnementaux majeurs liés à la désertification, la pollution sans oublier l'inondation de plus en plus récurrente dans la ville. Il est alors opportun de faire l'état des lieux de la flore et de la végétation de la ville de Malanville, non seulement située à la porte du sahel, mais aussi une ville commerciale abritant le deuxième plus grand marché du Bénin. La disponibilité d'une littérature sur l'état des lieux de la végétation urbaine aidera à une meilleure prise de décision au niveau locale et servira de tremplin à la promotion de la foresterie urbaine.
L'hypothèse qui sous-tend la présente recherche est que la ville de Malanville regorge d'une importante biodiversité floristique.

L'objectif de la présente recherche était de caractériser la végétation ligneuse à travers sa diversité et sa structure dans la ville de Malanville.

\section{MATERIEL ET METHODES Milieu d'étude}

La ville de Malanville, cadre de la présente recherche est située entre $11^{\circ} 50^{\prime} 38^{\prime}$ ' et $11^{\circ} 52^{\prime} 02^{\prime \prime}$ de latitude nord et entre $3^{\circ} 22^{\prime} 00^{\prime}$ 'et $3^{\circ} 24^{\prime} 02$ ', de longitude est. Elle correspond à l'arrondissement central de la Commune de Malanville localisée à l'extrême Nord de la République du Bénin, dans le département de l'Alibori, en bordure du fleuve Niger. Elle couvre une superficie de 945 ha (Figure 1).

Les caractéristiques biophysiques du milieu que sont : le climat, la température, les précipitations, l'insolation, le vent et le sol, conditionnent la plantation et la croissance de la végétation ligneuse urbaine. La ville de Malanville est sous l'influence du climat soudano-sahélien à deux saisons. Ce climat est caractérisé dans l'ensemble par des températures variant entre $18{ }^{\circ} \mathrm{C}$ et $45^{\circ} \mathrm{C}$ avec des amplitudes thermiques très élevées. Dans la ville, les précipitations sont irrégulières et varient d'une année à l'autre. Les hauteurs de pluie oscillent annuellement entre 800 et 950 mm (ASECNA, 2016). La saison des pluies dure entre cinq et six mois (juin à octobre) et la saison sèche (novembre à mai). La faiblesse des précipitations de la ville ne favorise pas le développement normal des arbres. L'insolation est le facteur principal de la photosynthèse et favorise la production de biomasse. Sa durée annuelle dans le secteur d'étude est en moyenne de 3000 heures. Elle est maximale en novembre $(286 \mathrm{~h})$ et minimale en août (184 h). L'alizé maritime (mousson) et l'alizé continental (harmattan) sont les deux types de vents dominants de la ville de Malanville. L'harmattan est un vent sec qui souffle de novembre à mars suivant une direction nord-est et sud-ouest. Sa vitesse moyenne décroît de 3 $\mathrm{m} / \mathrm{s}$ en avril à $2 \mathrm{~m} / \mathrm{s}$ en novembre. Sa vitesse 
maximale atteint $14 \mathrm{~m} / \mathrm{s}$ en janvier. Il augmente le déficit de saturation de l'air et accentue les conditions d'aridité de la saison sèche, donc empêche le développement normal des arbres et leur impose le repos végétatif (état de dormance). Enfin, le sol est le support des plantes et joue le rôle d'alimentation en air, en eau et en éléments minéraux nécessaires au bon développement des arbres. Les sols de Malanville sont sableux et hydromorphes pour la plupart. Ces types de sol sont favorables au développement des plantes du fait de leur horizon humifère et leur texture sableux facilitant l'infiltration de l'eau à condition qu'il y ait une forte précipitation bien répartie. On y rencontre dans la ville, la végétation ligneuse et herbacée sous diverses formes (isolée, alignée et groupée) dans les concessions, les institutions, les rues, les places publiques et les plantations forestières urbaines.

Les principales activités des populations de Malanville sont l'agriculture, l'élevage et le commerce. L'agriculture souffre $\mathrm{du}$ manque de terre cultivable. Elle est extensive et nécessite la destruction de la végétation au profit des terres cultivables chaque année. L'élevage occupe une place de choix dans la ville de Malanville. Toutefois la divagation des animaux (grands et petits ruminants) constitue un problème majeur pour la viabilité et le développement des espèces végétales urbaines. Le commerce est développé à Malanville et constitue la principale fonction dans la hiérarchie urbaine. En effet, la ville a su profiter de sa position géographique et ériger un marché international très fréquenté, le deuxième du Bénin après celui de Dantokpa (Cotonou), en termes de transactions (Sougue, 2016). Toutes ces activités imposent à la végétation urbaine des contraintes qui entravent son développement normal.

\section{Matériel}

Il comprend le matériel d'étude (végétation ligneuse) puis le matériel de collecte et de traitement des données. Comme matériel de collecte utilisé, il y a : un récepteur GPS (Global Positioning System) pour rallier les placeaux et prendre les coordonnées géographiques des arbres; une fiche d'inventaire pour noter les données collectées ; un penta décamètre pour mesurer la circonférence des arbres puis la distance de visée; un clinomètre pour la mesure des hauteurs des arbres; des papiers journaux pour la réalisation de l'herbier. Le matériel de traitement des données et d'analyse statistique comprend: le tableur Excel pour la saisie et l'analyse des données ; le logiciel ArcGIS 10.5 pour la cartographie; les logiciels SPSS et SAS 9.2 pour les analyses statistiques.

\section{Méthodes de collecte des données}

L'inventaire floristique a été réalisé dans 300 placeaux (Figure 2) installés suivant un échantillonnage aléatoire stratifié (Nowak et al., 2008; Nero et al., 2018). Un maillage systématique (Tuo et al., 2017) de la carte de forme carrée (Kim et al., 2015) de 100 m x 100 $\mathrm{m}$ (Chabi et al., 2016), soit 1 ha est fait. Les mailles (placeaux) sont numérotées de 1 à $n$ (Kim et al., 2015) selon la superficie en ha de la ville. Après quoi les points d'échantillonnage sont générés au hasard par le logiciel ArcGIS 10.5 sur la carte de la ville de Malanville, suivant la couverture végétale et la taille des arbres par strate. Le nombre total de placeaux installés est obtenu par la formule de Dagnelie (1998), après installation aléatoire de 10 placeaux pré-échantillon.

$$
\left.n_{t=\left[t_{1-\alpha / 2}\right.}(c v / d)\right]^{2}
$$

où $\mathrm{n}_{\mathrm{t}}$ : nombre total de placeaux; $\mathrm{cv}=$ 86,6 : coefficient de variation de la surface terrière ; $\mathrm{d}=10 \%$ : marge d'erreur d'estimation de la surface terrière avec $5 \leq \mathrm{d} \leq 15 \% ; t_{1-\frac{\alpha}{2}}$ : valeur de la statistique $\mathrm{t}$ de la distribution de Student pour un risque alpha de 0,$05 ; t_{1-\frac{\alpha}{2}} \approx$ 2

La Figure 2 présente la répartition spatiale des placeaux par unité d'occupation des terres. L'analyse de cette Figure révèle que les placeaux sont répartis sur toutes les unités d'occupation des terres identifiées dans la ville à l'exception des plans d'eau qui sont des zones difficiles d'accès.

Dans la ville de Malanville huit unités d'occupation des terres ont été identifiées suivant la classification de niveaux I et II faite 
par Anderson et al. (1976). Cette classification stipule que les types de catégorisation de l'utilisation et de la couverture des terres sont liés à la capacité des terres, à leur vulnérabilité à certaines pratiques de gestion et du potentiel pour une activité ou une valeur particulière des terres, qu'elle soit intrinsèque ou spéculative (Tableau 1).

Les données dendrométriques telles que la circonférence, la hauteur totale, la hauteur fût, les diamètres nord-sud et est-ouest des arbres ont été collectés.

\section{Méthodes de traitement des données floristiques}

Le tableur Excel a été utilisé pour la saisie et le traitement des données collectées. L'identification des espèces et la détermination de leur origine (endogène ou exogène) a été réalisé à l'aide de la flore analytique du Bénin (Akoegninou et al., 2006) et Arbres et arbustes du Sahel (Arbonnier, 1990). Les différents paramètres calculés sont :

\section{Richesse spécifique $(R)$}

C'est le nombre d'espèces présentes sur une aire déterminée de la ville de Malanville.

\section{Indice de diversité de Shannon $(H)$}

L'indice de diversité de Shannon varie à la fois en fonction du nombre d'espèces présentes et en fonction de la proportion relative des individus de diverses espèces. Il s'exprime en bits et varie généralement de 0 à 5. L'analyse des résultats est faite suivant les modalités utilisées par Logbo et al. (2020); $\mathrm{Si}$ $H \in] 0 ; 3$ [ $H$ est faible ; Si $H \in[3 ; 4[H$ est moyen et si $H \in[4 ;+[H$ est élevé. Il est d'autant plus élevé qu'un grand nombre d'espèces participe au recouvrement. C'est-àdire plus sa valeur est élevée plus la diversité est grande. Il se calcule suivant la formule :

$$
\mathrm{H}=-\sum p_{i} \log _{2} p_{i}
$$

$P i$ (compris entre 0 et 1): proportion relative de l'effectif des individus d'une espèce $i$ dans l'ensemble des individus de toutes les espèces concernées; $P i=n_{i} \sum n_{i}$; avec $n_{i}$ : effectif des individus de l'espèce $i$ et $\sum n_{i}:$ ensemble des individus de toutes les espèces.

\section{Equitabilité de Pielou (E)}

L'équitabilité traduit le degré de diversité atteint par un peuplement, et correspond au rapport entre la diversité effective $(\mathrm{H})$ et la diversité maximale théorique $\left(H_{\max }\right)$.

$$
E=H / H \text { max avec } H \text { max }=\log _{2} S
$$

$\mathrm{H}$ : indice de diversité de Shannon ; Hmax = $\log 2 \mathrm{~S}$ : valeur théorique de la diversité maximale pouvant être atteinte dans chaque groupement ; $\mathrm{S}$ : nombre total d'espèces.

L'équitabilité varie entre 0 et 1 . Elle tend vers 0 quand la quasi-totalité des effectifs correspond à une seule espèce du peuplement, et tend vers 1 lorsque chacune des espèces est représentée par le même nombre d'individus.

\section{Surface terrière moyenne (Gi)}

La surface terrière moyenne est la somme des sections des troncs des arbres à un niveau de référence. Elle est calculée à partir de la formule suivante :

$$
\mathbf{G i}=\mathbf{\Sigma} \mathbf{C}^{2} \times \mathbf{1 0 0 0 0 / 4 \Pi S}
$$

$\mathrm{C}$ : circonférence à $1,30 \mathrm{~m}$ du sol ; $\mathrm{S}$ : superficie inventoriée rapportée à l'hectare ; $\mathrm{Gi}$ : surface terrière en $\mathrm{m}^{2} / \mathrm{ha}$.

\section{Densité (D)}

Elle permet de connaître le nombre d'arbres de la ville rapportée à l'hectare, suivant la formule : $\quad \mathbf{D}=\mathbf{N} \times \mathbf{1 0 0 0 0 / S}$ $\mathrm{N}$ : nombre de tiges ayant au moins $1,30 \mathrm{~m}$ de hauteur; $\mathrm{S}$ : superficie inventoriée rapportée à l'hectare; D : nombre de tiges par hectare.

\section{Indices de valeur d'importance des espèces}

L'indice de valeur d'importance (IVI) a été calculé en utilisant les dérivés de la densité, de la surface terrière et de la fréquence de chaque espèce enregistrée pour caractériser la structure du peuplement (Adam et al., 2014). L'IVI varie de 0 à 300 . Les espèces dont la valeur de l'IVI est supérieure ou égale à 10 ont été considérées comme espèces écologiquement importantes dans le secteur d'étude. Sa formule est la suivante :

$\mathbf{I V I}=\mathbf{N i} / \mathbf{\Sigma} \mathbf{N i}+\mathbf{G i} / \mathbf{\Sigma} \mathbf{G i}+\mathbf{F i} / \mathbf{L F i}$ avec $\mathbf{F i}=\mathbf{r i} / \mathbf{R}$ $\mathrm{Ni}$ : nombre d'individus de l'espèce $\mathrm{i}$; Gi : surface terrière des individus de l'espèce $\mathrm{i}$; ri : nombre de relevés dans lequel l'espèce i est présentée ; $\mathrm{R}$ : nombre de relevés total. 


\section{Analyses statistiques}

Afin de caractériser le secteur d'étude, une analyse factorielle des correspondances a été réalisée avec le logiciel SPSS. Le logiciel Statistical Analysis System version 9.2 (SAS v. 9.2) a été utilisé pour les analyses statistiques. Ainsi, l'analyse de variance des différents paramètres ont été testé en utilisant la procédure GLM (General Linear Model) du logiciel SAS (ANOVA) ou le test de KruskalWallis. Le test de la plus petite différence significative (ppds) a été aussi utilisé pour la séparation des moyennes au seuil de 5\% (Dagnelie, 1986).

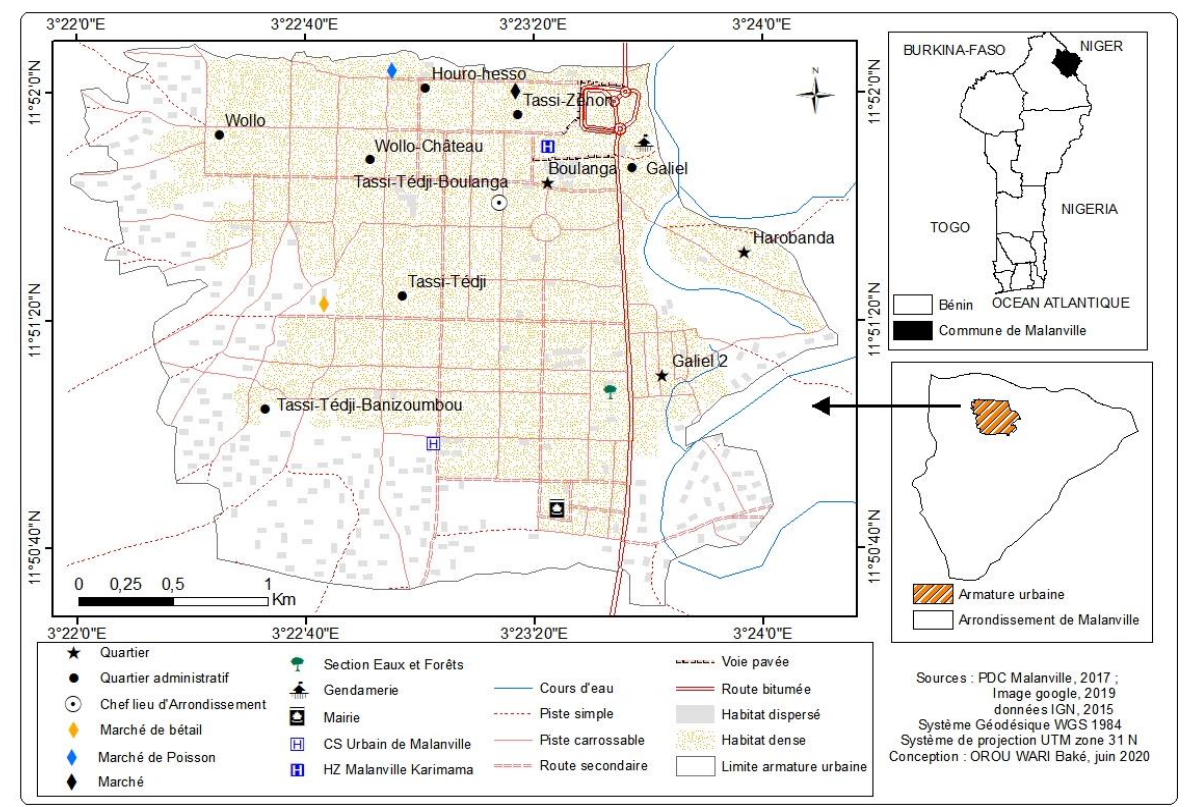

Figure 1 : Situations géographique et administrative de la ville de Malanville.

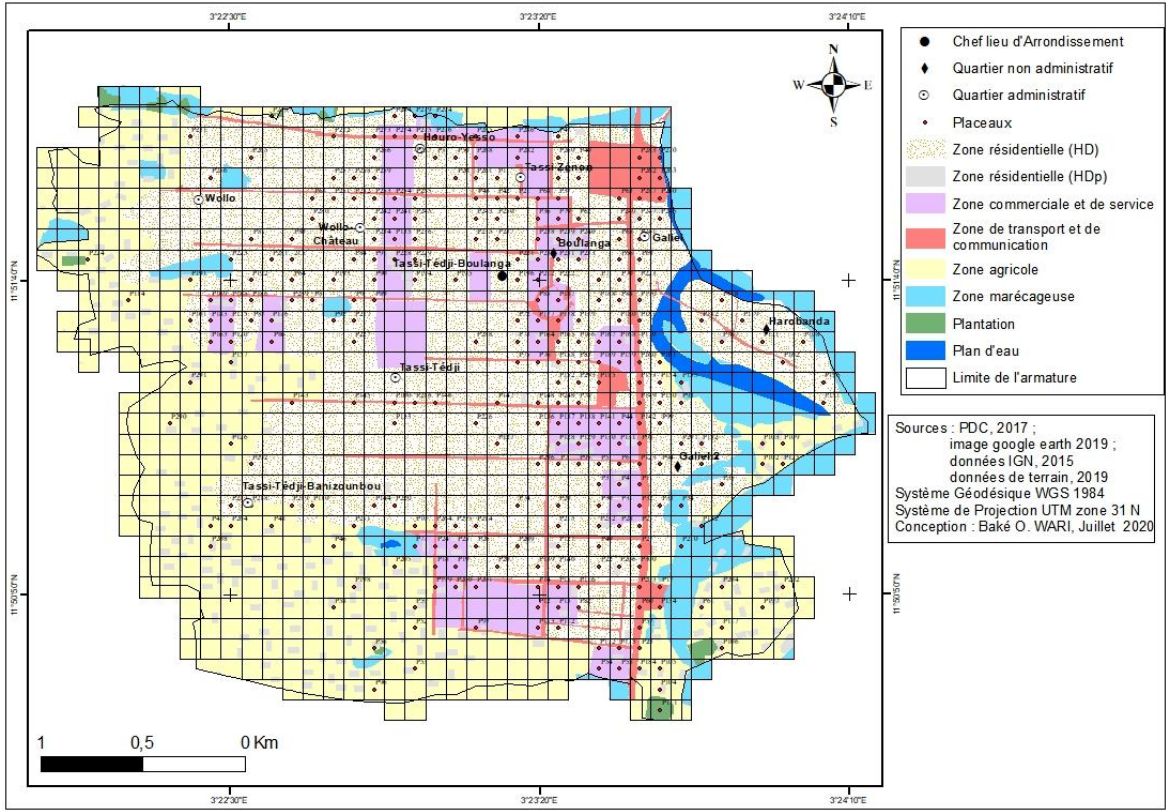

Figure 2 : Répartition spatiale des placeaux par unité d'occupation des terres. 
Tableau 1 : Système de classification de l'occupation des terres.

\begin{tabular}{|c|c|c|}
\hline Niveau I & Niveau II & Critères/éléments importants \\
\hline \multirow{7}{*}{$\begin{array}{l}1 \text { Terres } \\
\text { urbaines } \\
\text { ou bâti }\end{array}$} & 1.1 Résidentiel & Densité du bâti (habitat dense et dispersé) \\
\hline & $\begin{array}{l}1.2 \text { Commerce } \\
\text { et services }\end{array}$ & $\begin{array}{l}\text { Vente de produits et de services (bâtiments de } \\
\text { bureaux et d'institution, des entrepôts, des hangars, } \\
\text { établissements religieux, sanitaires, éducatifs, } \\
\text { correctionnels et militaires). }\end{array}$ \\
\hline & 1.3 Industrie & Industrie légère à industrie lourde \\
\hline & $\begin{array}{l}1.4 \text { Transports, } \\
\text { communications } \\
\text { et services } \\
\text { publics }\end{array}$ & Voies de transport, gares, parkings, rotondes \\
\hline & $\begin{array}{l}1.5 \text { complexes } \\
\text { industriels et } \\
\text { commerciaux }\end{array}$ & Parc industriel \\
\hline & $\begin{array}{ll}1.6 & \text { Terrains } \\
\text { urbains } & \text { ou bâtis } \\
\text { mixtes }\end{array}$ & Mélange d'utilisations de niveau II \\
\hline & $\begin{array}{lr}1.7 & \text { Autres } \\
\text { terrains } & \text { urbains } \\
\text { ou bâtis } & \end{array}$ & $\begin{array}{l}\text { Terrains de golf, zoos, parcs urbains, cimetières, } \\
\text { décharges, structures de contrôle des eaux et des } \\
\text { déversoirs, }\end{array}$ \\
\hline 2 Terres agricoles & - & Production de nourriture et de fibres \\
\hline 3 Terres de parcours & - & $\begin{array}{l}\text { Végétation naturelle principalement composée } \\
\text { d'herbes, de plantes herbacées ou d'arbustes }\end{array}$ \\
\hline 4 Terres forestières & - & $\begin{array}{l}\text { Fermeture couronne de } 10 \% \text {, moins de } 10 \% \text { fermeture } \\
\text { couronne mais non aménagées pour d'autres usages et } \\
\text { plantation en bloc de la catégorie urbaine ou bâtie }\end{array}$ \\
\hline \multirow[t]{2}{*}{5 Eau } & - & Cours d'eau, plans d'eau \\
\hline & - & $\begin{array}{l}\text { Dépressions topographiques (marais, vasières et } \\
\text { marécages) }\end{array}$ \\
\hline 7 Terre stérile & - & Zone de terre mince, de sable ou de roches \\
\hline
\end{tabular}

Source : Anderson et al., 1976 / recherche documentaire.

\section{RESULTATS}

\section{Caractéristiques des unités d'occupation des terres de la ville de Malanville}

Dans la ville de Malanville la végétation ligneuse est présente dans les zones résidentielles, les zones commerciales et de service, les zones de transport et de communication, les zones agricoles et les zones marécageuses, mais sous forme des arbres isolés ou plantation d'accompagnement, des arbres sur les voies de communication ou plantation d'alignement et des arbres groupés ou plantation forestière urbaine. La matrice brute des 68 espèces dénombrées et les sept unités d'occupation des terres identifiées dans la ville sont soumises à une analyse factorielle des correspondances. Le Tableau 2 présente le pourcentage de variance expliquée et les corrélations. L'analyse du Tableau 2 montre que les deux premiers axes factoriels expliquent $75 \%$ de l'inertie totale, donc ces deux axes peuvent être utilisés pour faire une bonne synthèse des informations. Le Tableau 2 révèle que les espèces et les unités d'occupation des terres sont bien représentées sur les deux axes avec une forte représentativité au niveau de l'axe 1. Le Tableau 3 présente la synthèse des corrélations entre les espèces et 
les unités de l'occupation des terres. De l'analyse du Tableau 3, il ressort que les unités d'occupation des terres urbaines de Malanville sont fortement corrélées avec les différentes espèces inventoriées. La Figure 3 présente la projection des espèces et des unités d'occupation des terres sur les deux axes. L'analyse de la Figure 3 monte que sur l'axe 1, les espèces telles que : Citrus sinensis (Csi), Acacia sieberi(Asi) Albizia lebbeck (Al) et Tamarindus indica (Tin) caractérisent les plantations dans la ville de Malanville. Tandis que sur l'axe 2 un grand nombre d'espèces dont les plus importantes sont: Moringa oleifera (Mo), Azadirachta indica (Ai), Ficus umbellata $(F u)$, Vitex doniana (Vdo, Ficus thonningii (Fth), Borassus aethiopum (Bae) sont plus représentées dans les unités d'occupation comme les zones résidentielles (ZR, ZR1), les zones de transport et de communication (ZTC) ; et les zones agricoles (ZA). Par contre, elles s'opposent aux zones marécageuses (ZM) puis de commerce et de service dont les espèces caractéristiques sont Eucalyptus camaldulensis (Eca), Acacia auriculiformis (Aa), Bauhinia rufescens $(\mathrm{Br})$ et Citrus maxima (Cm).

\section{Diversité floristique et paramètres structuraux de la végétation ligneuse urbaine \\ Composition floristique et diversité spécifique de la végétation urbaine ligneuse de Malanville}

Au total, 6108 individus répartis en 68 espèces, 58 genres et 33 familles ont été recensés dans les 300 placeaux. Ces espèces sont pour la plupart introduites (61\% exotiques contre $39 \%$ endogènes). Les familles les mieux représentées sont les LeguminosaeCaesalpinioideae (7 espèces, 6 genres), les Arecaceae (5 espèces, 5 genres); les Bignoniaceae (5 espèces, 5 genres); les Moraceae (5 espèces, 1 genre) et les Leguminosae-Mimosoideae (4 espèces, 3 genres). Le nombre total d'espèces inventoriées et la diversité floristique de la végétation en milieu urbain à Malanville relativement faible varient selon les types de végétation dans la ville (Tableau 4). L'analyse du Tableau 4 montre que dans la ville de Malanville les zones résidentielles ont enregistré le plus grand nombre d'espèces végétales, tandis que le plus petit (12) nombre d'espèces est enregistré dans les zones marécageuses. Les résultats de l'analyse de variance des paramètres de diversité révèlent des différences significatives (Tableau 4) entre les unités d'occupation des terres en termes de richesse spécifique $(\operatorname{Pr}=0,0016)$ et de l'indice de diversité de Shannon $(\operatorname{Pr}=0,0177)$, à l'exception de l'équitabilité de Pielou ( $\mathrm{Pr}=$ 0,3526). De l'analyse comparative, il ressort que les plus fortes valeurs de richesse spécifique et d'indice de diversité de Shannon (H) s'observent au niveau des plantations tandis que les plus faibles valeurs sont enregistrées au niveau des zones agricoles. Par contre, l'équitabilité de Pielou reste statistiquement similaire entre les unités d'occupation des terres et montrent que la répartition des individus par espèce est régulière dans toutes les unités d'occupation des terres, à l'exception des zones marécageuses qui ont enregistré la plus faible valeur. Globalement, les paramètres de diversité témoignent d'une répartition régulière des espèces dans les zones résidentielles.

\section{Paramètres structuraux de la végétation urbaine ligneuse}

La densité, la surface terrière et la circonférence varient par unité d'occupation et présentent des valeurs faibles. La Figure 4 présente les paramètres de structure de la végétation ligneuse urbaine de Malanville. L'analyse de la Figure 4 révèle que la densité et la surface terrière varient de manière significative entre les différentes unités d'occupation des terres, mais la circonférence moyenne ne varie pas. Les valeurs de la densité et de la surface terrière décroissent significativement $(\mathrm{p}<0,01)$ des plantations forestières vers les autres unités d'occupation des terres, avec les plus faibles valeurs enregistrées au niveau des zones agricoles. Par ailleurs la circonférence moyenne varie de $90,7 \pm 14,81$ dans les plantations à $121,17 \pm 12,39 \mathrm{~cm}$ dans les zones résidentielles. Ce résultat peut s'expliqué par une existence de compétition entres les arbres au niveau des 
plantations. L'analyse de toutes les caractéristiques dendrométriques des unités d'occupation des terres étudiées permet de déduire que les plus gros arbres se trouvent dans les zones résidentielles suivi des zones commerciales et de service, des zones de transport et de communication, des zones agricoles et des zones marécageuses, avec moins d'arbres à l'hectare, tandis que les plantations ont plus d'arbres à l'hectare mais avec de petites circonférences.

\section{Importance écologique des espèces ligneuses de la végétation urbaine}

L'Indice de Valeur d'Importance des espèces (IVI) caractérise l'importance au sein d'une végétation, d'une espèce par rapport à l'ensemble de toutes les espèces présentes dans la végétation considérée. Le Tableau 5 présente les espèces écologiquement importantes (IVI $\geq$ 10) par unité de végétation. La lecture du Tableau 5 montre les cinq (5) espèces dont les valeurs d'importance (IVI) sont les plus élevées dans la ville de Malanville. De l'analyse du Tableau 5, il ressort que Azadirachta indica (160) est l'espèce végétale la plus écologiquement importante dans la ville de Malanville. Elle est suivie de Eucalyptus camaldulensis (38), de Terminalia mantaly (21), de Ficus polita (12) et de Gmelina arborea (12). Au total, les cinq espèces écologiquement importantes concentrent $81,44 \%$ de la valeur totale d'importance de l'ensemble des espèces inventoriées dans la ville et Azadirachta indica en concentre à elle seule $53,61 \%$.

Tableau 2 : Pourcentage de variance expliquée et les corrélations.

\begin{tabular}{llcc}
\hline & \multicolumn{2}{c}{ Axe } & \\
\cline { 2 - 3 } & 1 & $\mathbf{2}$ & Moyenne \\
\hline Espèces & 0,791 & 0,709 & 0,750 \\
Unités d'occupation & 0,791 & 0,709 & 0,750 \\
Total actif & 1,582 & 1,419 & 1,500 \\
Pourcentage de variance & 79,089 & 70,935 & 75,012 \\
expliquée & & & \\
\hline
\end{tabular}

Source : Travaux de terrain, Malanville 2019.

Tableau 3 : Corrélations entre les espèces et les unités d'occupation des terres.

\begin{tabular}{lcc}
\hline & Espèces & Unités d'occupation \\
\hline Espèces & 1,000 & 0,582 \\
Unités d'occupation & 0,582 & 1,000 \\
Dimension & 1 & 2 \\
Valeur propre & 1,582 & 0,418 \\
\hline
\end{tabular}

Source : Travaux de terrain, Malanville 2019. 


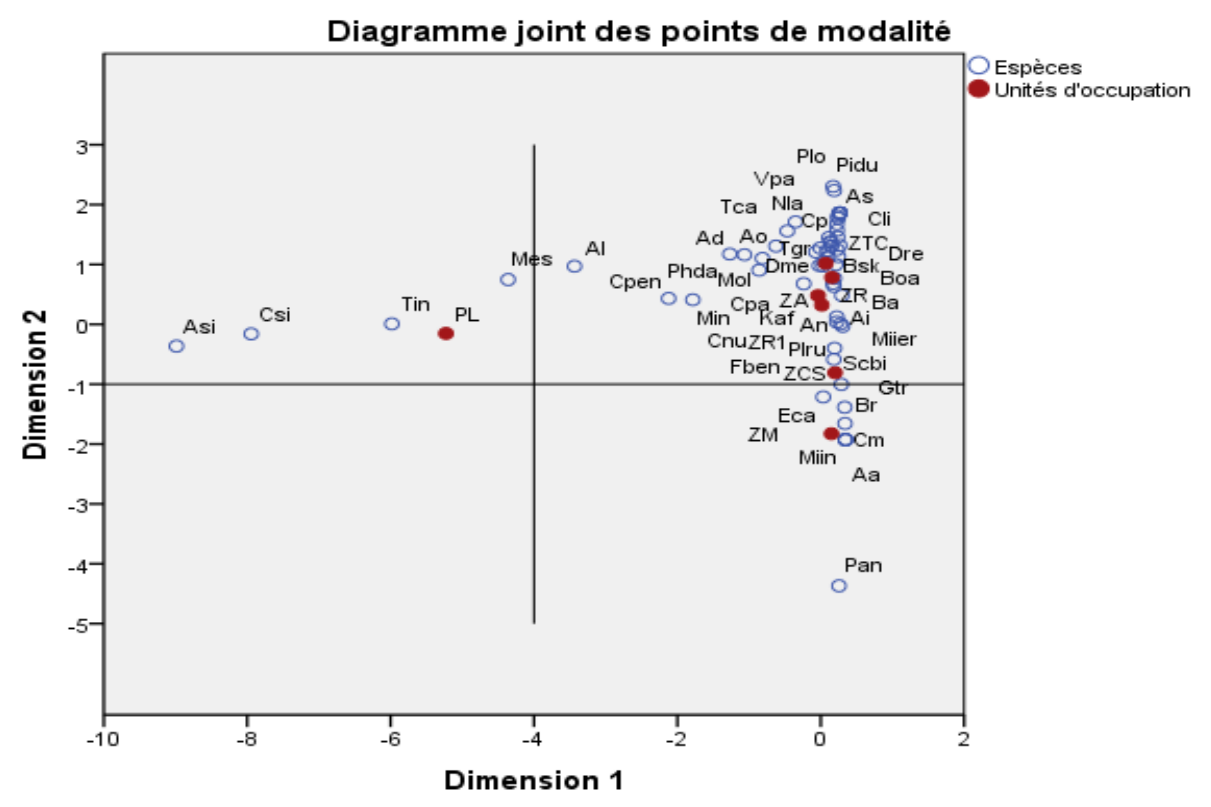

Normalisation principale de la variable.

Figure 3 : Projection des espèces et des unités d'occupation des terres sur les axes.

Tableau 4 : Paramètres de diversité floristique de la végétation ligneuse urbaine de Malanville.

\begin{tabular}{|c|c|c|c|c|c|}
\hline Type de végétation & Nombre d'espèce & & R (espèces) & H' (bit) & $\mathbf{E}$ \\
\hline \multirow{3}{*}{ ZR } & & $\mathrm{m}$ & $4,39^{\mathrm{b}}$ & $1,37^{\mathrm{ab}}$ & $0, \mathbf{6 3}^{\mathrm{a}}$ \\
\hline & 53 & $\sigma$ & 0,23 & 0,08 & 0,03 \\
\hline & & $\mathrm{m}$ & $3,89^{\mathrm{b}}$ & $1,26^{\mathrm{ab}}$ & $0,63^{\mathrm{a}}$ \\
\hline \multirow{2}{*}{ ZR1 } & 37 & $\sigma$ & 0,44 & 0,15 & 0,06 \\
\hline & & $\mathrm{m}$ & $3,31^{\mathrm{b}}$ & $0,96^{\mathrm{b}}$ & $0,53^{\mathrm{a}}$ \\
\hline $\mathrm{ZCS}$ & 34 & $\sigma$ & 0,30 & 0,1 & 0,05 \\
\hline ZTC & 24 & $\begin{array}{l}\mathrm{m} \\
\sigma\end{array}$ & $\begin{array}{c}2.87^{\mathrm{b}} \\
0,43\end{array}$ & $\begin{array}{c}0.91^{\mathrm{b}} \\
0,18\end{array}$ & $\begin{array}{c}0.52^{\mathrm{a}} \\
0,08\end{array}$ \\
\hline $\mathrm{ZA}$ & 21 & $\begin{array}{c}\mathrm{m} \\
\sigma\end{array}$ & $\begin{array}{c}2,75^{\mathrm{b}} \\
0,42\end{array}$ & $\begin{array}{c}1,04^{\mathrm{ab}} \\
0,21\end{array}$ & 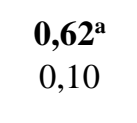 \\
\hline \multirow[t]{2}{*}{$\mathrm{ZM}$} & \multirow[t]{2}{*}{20} & $\mathrm{~m}$ & $0,91^{\mathrm{b}}$ & $0,83^{\mathrm{b}}$ & $0,48^{\mathrm{a}}$ \\
\hline & & $\sigma$ & 0.55 & 0,2 & 0,1 \\
\hline \multirow[t]{2}{*}{ PL } & \multirow[t]{2}{*}{12} & $\mathrm{~m}$ & $6,75^{\mathrm{a}}$ & $1,64^{\mathrm{a}}$ & $0,59^{\mathrm{a}}$ \\
\hline & & $\sigma$ & 2,06 & 0,58 & 0,11 \\
\hline Probabilité & - & - & $\mathrm{p}<\mathbf{0 , 0 1}$ & $\mathrm{p}<0,05$ & p > 0,05 \\
\hline
\end{tabular}

Source : Travaux de terrain, Malanville 2019.

Richesse spécifique (R), Indice de Shannon (H'), Equitabilité de Pielou (E), ZR : Zone résidentielle à habitation dense, ZR1 : Zone résidentielle à habitation dispersée, ZCS : Zone commerciale et de services, ZTC: Zone de transport et de communication, ZA : Zone agricole, ZM : Zone marécageuse, PL : plantation. Les valeurs accompagnées de la même lettre ou de même combinaison de lettre(s) (a, b ou c) ne sont pas significativement différentes au seuil de 5\% (test de structuration des valeurs moyennes de Scheffé). 


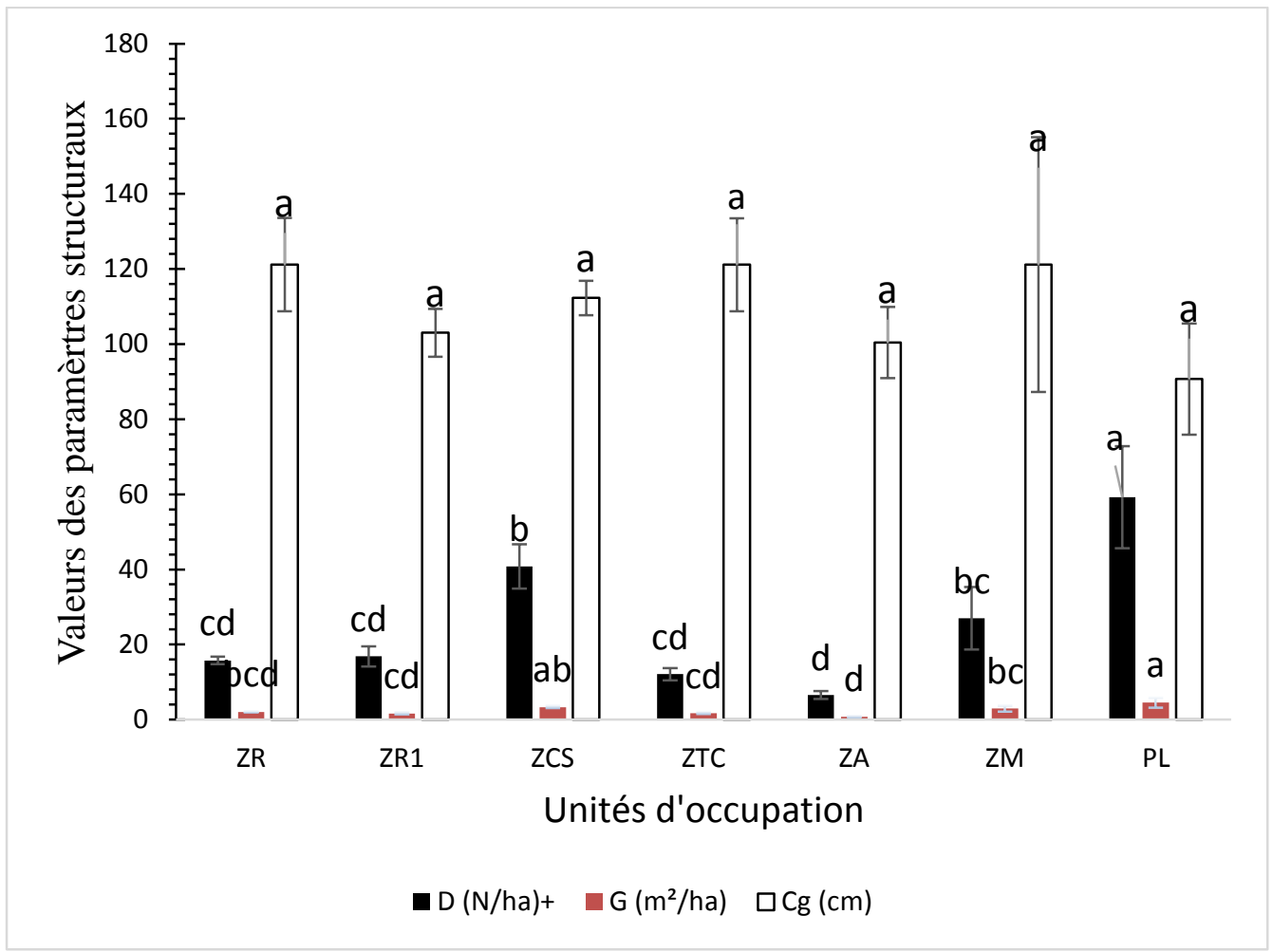

$\mathrm{D}(\mathrm{N} / \mathrm{ha})$ : Densité ; G (m²/ha) : Surface terrière ; $\mathrm{Cg}(\mathrm{cm})$ : Circonférence moyenne ; ZR : Zone Résidentielle à habitation dense ; ZR1 : Zone résidentielle à habitation dispersée ; ZCS : Zone Commerciale et de Services ; ZTC : Zone de Transport et de Communication ; ZA : Zone Agricole ; ZM : Zone Marécageuse ; PL : Plantation. Les valeurs accompagnées de la même lettre ou de même combinaison de lettre(s) (a, b ou c) ne sont pas significativement différentes au seuil de 5\% (test de structuration des valeurs moyennes de Scheffé).

Figure 4 : Paramètres de structure de la végétation urbaine de Malanville.

Tableau 5 : Espèces écologiquement importantes (IVI $\geq 10$ ) par unité de végétation.

\begin{tabular}{|c|c|c|c|c|c|c|c|c|}
\hline Espèces & ZR & ZR1 & ZCS & ZTC & $\mathbf{Z A}$ & $\mathbf{Z M}$ & PL & Global \\
\hline $\begin{array}{l}\text { Azadirachta } \\
\text { indica }\end{array}$ & 67,75 & 12,25 & 63,89 & 9,29 & 2,69 & 4,25 & 0,73 & 160,84 \\
\hline $\begin{array}{c}\text { Eucalyptus } \\
\text { camaldulensis }\end{array}$ & 6,92 & 3,40 & 21,30 & 0,30 & 0,47 & 5,05 & 1,02 & 38,47 \\
\hline Ficus polita & 8,34 & 1,49 & 1,40 & 0,08 & 0,58 & 0,00 & 0,00 & 11,89 \\
\hline $\begin{array}{l}\text { Gmelina } \\
\text { arborea }\end{array}$ & 6,44 & 2,00 & 2,13 & 0,79 & 0,12 & 0,00 & 0,24 & 11,72 \\
\hline $\begin{array}{l}\text { Terminalia } \\
\text { mantaly }\end{array}$ & 11,59 & 2,80 & 4,74 & 1,17 & 0,23 & 0,70 & 0,16 & 21,39 \\
\hline Total & 10,04 & 21,94 & 93,46 & 11,63 & 4,09 & 10,00 & 2,15 & 244,31 \\
\hline
\end{tabular}




\section{DISCUSSION}

\section{Composition floristique et diversité spécifique de la végétation urbaine de Malanville}

Sur l'ensemble des relevés 68 espèces réparties en 58 genres et 33 familles ont été inventoriées. Ainsi, le nombre d'espèces recensées à Malanville est proche à celui obtenu par Amontcha (2018) dans les espaces verts publics de la ville de Cotonou (66 espèces) et de Porto-Novo (73 espèces), mais, l'auteur lors de sa recherche, a pris en compte toutes les espèces végétales. Par ailleurs, dans une recherche intitulée « Structure, diversité et stocks de carbone des arbres dans la ville de Kumasi au Ghana », Nero et al. (2018) ont obtenu 3757 arbres et arbustes regroupés en 176 espèces et 42 familles dans les différents types d'espaces verts urbains de la ville de Kumasi. Ces résultats montrent une grande diversité des espèces végétales dans la ville de Kumasi contrairement à celle de Malanville qui enregistre plus d'arbres, mais moins diversifiés. Au Togo, des valeurs élevées aux nôtres ont été aussi enregistrées (Radji et al., 2010 ; Polorigni et al., 2014) dans la ville de Lomé respectivement (612 espèces et 72 familles; 93 espèces et 47 familles). Les faibles valeurs de diversité de la végétation urbaine de Malanville s'expliquent par les conditions climatiques et édaphiques peu favorable à la survie de plusieurs espèces végétales. A cela s'ajoutent la divagation des animaux et l'absence des initiatives locales de plantation et de conservation des arbres.

\section{Paramètres Structuraux et importance écologique de la végétation urbaine de Malanville}

Les fortes valeurs de la densité et de la surface terrière respectivement 59,25 $\pm 13,62$ arbres/ha et 4,52 $\pm 1,34 \mathrm{~m}^{2} / \mathrm{ha}$ sont observées au niveau des plantations forestières. Ensuite viennent respectivement les zones commerciales et de services $(40,78 \pm 5,92$ arbres/ha et $3,34 \pm 0,31 \mathrm{~m}^{2} / \mathrm{ha}$ ) et les zones marécageuses $(27 \pm 8,35$ arbres/ha et 2,96 \pm $0,79 \mathrm{~m}^{2} / \mathrm{ha}$ ). Par contre, une différence non significative de densité et de surface terrière est enregistrée au sein des trois unités d'occupation des terres urbaines: zones résidentielles $1(16,86 \pm 2,67$ arbres/ha et $1,65 \pm 0,28 \mathrm{~m}^{2} / \mathrm{ha}$ ), zones résidentielles $\left(15,75 \pm 1,02\right.$ arbres/ha et $\left.2,04 \pm 0,13 \mathrm{~m}^{2} / \mathrm{ha}\right)$, zones de transport et de communication $\left(12,09 \pm 1,66\right.$ arbres/ha et $\left.1,75 \pm 0,18 \mathrm{~m}^{2} / \mathrm{ha}\right)$. Les faibles valeurs des paramètres de structure sont enregistrées au niveau des zones agricoles. Ces faibles valeurs au niveau des zones agricoles s'expliquent par le fait que dans la ville, les impacts de la désertification sont visibles car des parcelles vides sont dépourvus des arbres et ce n'est qu'après l'installation des populations qu'ils plantent et entretiennent les arbres. En plus, les faibles valeurs se justifient par une saison de pluie plus courte avec de faible précipitation enregistrée par an sur un sol sableux à forte infiltration rendant ainsi difficile la plantation et l'entretien des arbres. Ces différents résultats corroborent ceux de Nero et al. (2018) dans la ville de Kumasi au Ghana qui ont observé moins d'arbres dans les prairies et les terres agricoles et plus d'arbres au niveau des institutions. Par contre, les résultats, obtenus par ces auteurs, selon lesquels, la richesse et la diversité des espèces sont nettement plus élevées dans la zone périurbaine que dans la zone urbaine centrale sont contraires aux résultats de la présente étude qui révèle que la zone urbaine centrale (zones résidentielles) est plus riche et diversifiée que la zone périurbaine (zones résidentielles 1). Ces résultats confirment le constat fait dans la ville où les espaces non habités sont dépourvus d'arbres.

Dans la ville de Malanville, Azadirachta indica concentre $53,61 \%$ de la valeur totale d'importance de l'ensemble des espèces inventoriées. Ce résultat explique que les conditions climatiques et édaphiques de Malanville sont favorables au développement de Azadirachta indica qui est une espèce très résistante. Ainsi, les résultats des travaux de Charahabil et al. (2018) sur la diversité et structure des espaces végétalisés urbains de la ville de Ziguinchor au Sénégal montre une fréquence relative de $90 \%$ de Azadirachta indica. Également dans la ville de N'Djaména, la FAO (2012) a recensé comme espèce 
dominante Azadirachta indica, avec 30\% inventoriées dans la zone urbaine et $37 \%$ dans la zone périurbaine. La ville de Malanville étant la porte d'entrée des pays sahéliens au Bénin, Azadirachta indica rend deux catégories de services très importantes, il s'agit de la régulation du microclimat urbain et du service culturel qui regroupe la notion de loisirs et de repos, source de bien-être des citadins. L'identification des cinq espèces écologiquement importantes (IVI $\geq 10$ ) montre l'absence quasi-totale des espèces fruitières à haute valeur socio-économique dans la ville de Malanville. Il serait intéressant que la journée de l'arbre à Malanville soit orientée de manière à créer des conditions favorables aux populations pour la plantation et l'entretiens des arbres.

\section{Conclusion}

$\mathrm{Au}$ terme de la présente recherche, on retient que, dans la ville de Malanville, la composition floristique et la diversité floristique varient de façon significative au sein des unités d'occupation des terres urbaines. Les résultats montrent que la ville de Malanville regorge d'une importante diversité floristique dominée par Azadirachta indica, espèce qui résiste et s'adapte mieux aux conditions climatiques et édaphiques de la ville. Les espaces urbanisés constituent donc un refuge important de la biodiversité végétale qu'il serait important de connaitre, de préserver, voire d'augmenter, afin d'optimiser leur potentiel d'atténuation des effets néfastes aux changements climatiques. Ces résultats permettent une meilleure connaissance de la diversité et la structure de la végétation urbaine de Malanville condition sine qua none d'inspirer l'action publique à s'engager pour la création, le maintien et la gestion durable des espaces végétalisés en milieu urbain.

\section{CONFLIT D'INTERETS}

Les auteurs déclarent qu'ils n'ont pas de conflit d'intérêt lié à cet article.

\section{CONTRIBUTIONS DES AUTEURS}

BOW est l'auteure principale de cette recherche, elle a participé à toutes les phases du travail. La validation de la méthodologie d'inventaire, du choix du site d'étude et la correction du manuscrit ont été effectués par SZ, MD, IY, BAHT, JGD sous la supervision de ITI encadreur principal de cette recherche.

\section{REFERENCES}

Adam T, Adamou MM, Chaibou I, Mahamane A. 2014. Structure et dynamique de la végétation ligneuse juvénile issue de la régénération naturelle assistée (RNA) dans le Centre-Sud du Niger. Int. J. Biol. Chem. Sci., 8(2): 649-665. DOI: http://dx.doi.org/10.4314/ijbcs.v8i2.22

Akoegninou A, Van der Burg WJ, Van der Maesen LJG. 2006. Flore Analytique du Bénin. Backhuys Publishers: Wageningen.

Amontcha AAM, Djègo JG, Lougbégnon TO, Sinsin BA. 2017. Typologie et répartition des espaces verts publics dans le grand Nokoué (Sud Bénin). European Scientific Journal, 13(21): 79-97. DOI: http://dx.doi.org/10.19044/esj.2017.v13n 21p79

Amontcha AAM. 2018. Typologie, utilités et contraintes à l'aménagement des espaces verts dans les villes du grand Nokoué (sud-Bénin). $\mathrm{PhD}$ thèse, Université d'Abomey-Calavi, Abomey-Calavi, 243 p.

Anderson JR, Hardy EE, Roach JT, Witmer RE. 1976. A Land Use and Land Cover Classification System for Use with Remote Sensor Data (ed). United States Government Printing Office: Washington.

Arbonnier M. 1990. Arbres et arbustes du Sahel: leurs Caractéristiques et leurs Utilisations. Verlag Josef Margraf: Paris.

Blanchart A, Sere G, Cherel J, Warot G, Stas M, Consales JN, Christophe Schwartz C. 2017. Contribution des sols à la production de services écosystémiques en milieu urbain. Environnement Urbain / Urban Environment, 11: 1916-4645. http://journals.openedition.org/eue/1809

Chabi A, Lautenbach S, Orekan VOA, Kyei-Baffour N. 2016. Allometric 
models and aboveground biomass stocks of a West African Sudan Savannah watershed in Benin. Carbon Balance Manage, 11(16): $18 \mathrm{p} . \quad$ DOI: http://dx.doi.org/10.1186/s13021-0160058-5

Charahabil MM, Cesar B, Hamadou B, Ndiaye S, Diatta M. 2018. Diversité et structure des espaces végétalisés urbains de la ville de Ziguinchor, Sénégal. Int. J. Biol. Chem. Sci., 12(4): 1650-1666. DOI: https://dx.doi.org/10.4314/ijbcs.v12i4.12

Curtis JT, Macintosh RP. 1950. The interrelations of certain analytic and synthetic phytosociological characters. Ecology, 31(3): 435-455. DOI: https://doi.org/10.2307/1931497

Dagnelie P. 1986. Théorie et Méthodes Statistiques: Applications Agronomiques, (Vol 2). Les presses agronomiques de Gembloux : Belgique.

Dagnelie P. 1998. Statistique Théorique et Appliquée, (Vol 2). De Boeck \& Larcier : Paris.

Djomo AN, Ibrahima A, Saborowski J, Gravenhorst G. 2010 Allometric equations for biomass estimations in Cameroon and pan moist tropical equations including biomass data from Africa. For. Ecol. Manage., 260: 18731885.

DOI:

http://dx.doi.org/10.1016/j.foreco.2010.0 8.034

FAO. 2012. Étude sur la foresterie urbaine et périurbaine de N'Djaména, Tchad: Rôle et place de l'arbre en milieu urbain et périurbain. FAO-Document de travail sur la foresterie urbaine et périurbaine, Rome.

Kim G, Miller PA, Nowak DJ. 2015. Assessing urban vacant land ecosystem services: Urban vacant land as green infrastructure in the City of Roanoke, Virginia. Urban Forestry \& Urban Greening, 8(679): 519526.

DOI: http://dx.doi.org/10.1016/j.ufug.2015.05. 003

Le Gourrierec S. 2012. L'arbre en ville : le paysagiste concepteur face aux contraintes du projet urbain. Sciences agricoles. Mémoire de fin d'étude Dumas-00739439, Université de Nantes, Nantes, p. 21

Henry M, Besnard A, Asante WA, Eshun J, Adu-Bredu S, Valentini R, Bernoux M, Saint-André L. 2010. Wood density, phytomass variations within and among trees, and allometric equations in a tropical rainforest of Africa. For. Ecol. Manage., 260: 1375-1388. DOI: http://dx.doi.org/10.1016/j.foreco.2010.0 7.040

Logbo J, Yedomonhan P, Tente Akoegninou A. 2020. Distribution et habitats de Newbouldia laevis (P.Beauv.) Seemann ex Bureau et de Dracaena arborea (Willd.) Link dans les zones bioclimatiques du Bénin. Int. J. Biol. Chem. Sci., 14(8): 2903-2927. DOI: https://dx.doi.org/10.4314/ijbcs.v14i8.20

Lougbégnon TO. 2013. Évaluation de la diversité des essences forestières urbaines de la ville de Porto-Novo et leurs utilisations par les populations locales, Revue de géographie du laboratoire Leïdi, 515(11) : 326-341.

Matsuoka RH, Kaplan R. 2014. People needs in the urban landscape: Analysis of Landscape And Urban Planning contributions. J. Landscape and Urban Planning, 84(1): 7-19. DOI: http://dx.doi.org/10.1016/j.landurbplan.2 007.09.009

Nero BF, Callo-Concha D, Denich M. 2018. Structure, Diversity, and Carbon Stocks of the Tree, Community of Kumasi, Ghana. Forests, 9(519): 17. DOI: http://dx.doi.org/10.3390/f9090519

Nero BF, 2017. Urban Green Spaces Enhance Carbon Sequestration and Conserve Biodiversity in Cities of the Global South: Case of Kumasi Ghana. Thèse de doctorat, University of Bonn: Bonn, Germany, $158 \mathrm{p}$.

Nowak DJ, Hoehn RE, Crane DE, Stevens JC, Walton JT. 2006. Assessing Urban Forest Effects and Values: Minneapolis' Urban Forest. USDA Forest Service, Northeastern Resource Bulletin, 166: 20 p.

DOI: 
http://dx.doi.org/10.1016/j.ufug.2006.01 . 007

Nowak DJ, Hoehn RE, Crane DE, Stevens JC, Walton JT, Bond J. 2008. A ground-based method of assessing urban forest structure and ecosystem services. Arboriculture and Urban Forestry, 34(6): 347-358. http://www.nrs.fs.fed.us/pubs/jrnl/2008/n rs_2008_nowak_001.pdf.

Pielou EC. 1966. Species diversity and pattern diversity in the study of ecological succession. J. Theor. Biol., 10: 370-383. DOI: http://dx.doi.org/10.1016/00225193(66)90133-0

PNUD. 2019. Planifier l'adaptation au changement climatique. PNUD. https://www.bj.undp.org/content/benin/fr /home/presscenter/articles/une-nouvelledynamique-au-processus-de-plannational-dadaptation.html

Osséni AA, Tohozin BAC, Toko MI, Sinsin B. 2014. Contribution des SIG dans l'analyse floristique des espaces verts dans la ville de Porto-Novo au Bénin. Rev. Ivoir. Sci. Technol., 23: 103-121. http://www.revist.ci

Polorigni B, Raoufo R, Kouami K. 2014. Perceptions, tendances et préférences en foresterie urbaine : cas de la ville de Lomé au Togo. European Scientific Journal, 10(5): 261-277. DOI: http://dx.doi.org/10.19044/ESJ.2014.V1 0N5P\%P
Radji R, Kokou K, Akpagana K. 2010. Etude diagnostique de la flore ornementale togolaise. Int. J. Biol. Chem. Sci., 4(2): 491-508.

DOI: https://doi.org/10.4314/ijbcs.v4i2.5 8159

Shannon CE, Weaver W, 1949. The Mathematical Theory of Communication. Univ. Illinois Press-Urbana : Chicago IIIUSA.

Selmi W, Weber C. 2017. Évaluation des services écosystémiques urbains : de la rhétorique à la pratique. L'apport de l'approche par habitat. Environnement Urbain, 11: 10p. DOI: https://doi.org/10.7202/1050495ar

Sougue E. 2016. Malanville - Gaya, une dynamique de territorialisation à la frontière Benin - Niger. Revue de Géographie et Aménagement, 29: 19505698.

DOI: https://doi.org/10.4000/tem.3216

Tuo FN, Koffi KJ, Kouassi AF, Kone M, Adama B, Bogaert J. 2017. Etude de la diversité, de l'endémisme et de la distribution spatiale des Rubiaceae de Côte d'Ivoire. Int. J. Biol. Chem. Sci., 11(2): 777-797. DOI: https://dx.doi.org/10.4314/ijbcs.v11i2.20 Yuanying P, Thomas S, Tianb D. 2008. Forest management and soil respiration: Implications for carbon sequestration. Environ. Rev., 16: 93-111. DOI:

DOI: https://doi.org/10.1139/A08-003 